\title{
Research Paper \\ The Mediating Role of Fear of Contagion in Explaining the Relationship Between Disgust Propensity and Fear of Contamination
}

\section{Zahra Zanjani ${ }^{1},{ }^{*}$ Hamid Yaghubi ${ }^{2}$, Ladan Fata ${ }^{3}$, Mohammadreza Shaiiri ${ }^{4}$, Mohammad Gholami Fesharaki ${ }^{5}$}

\author{
1. PhD in Clinical Psychology, Department of Clinical Psychology, Faculty of Medicine, Kashan University of Medical Sciences, Kashan, Iran . \\ 2. PhD in Clinical Psychology, Assistant Professor, Department of Psychology, Faculty of Humanities, Shahed University, Tehran, Iran. \\ 3. PhD in Clinical Psychology, Assistant Professor, Department of Clinical Psychology, School of Behavioral Sciences and Mental Health (Tehran Institute of Psychiatry), \\ Iran University of Medical Sciences, Tehran, Iran. \\ 4. PhD in Psychology, Associate Professor, Department of Psychology, Faculty of Humanities, Shahed University, Tehran, Iran. \\ 5. PhD in Biostatistics, Assistant Professor, Department of Biostatistics, School of Medical Sciences, Tarbiat Modares University, Tehran, Iran.
}

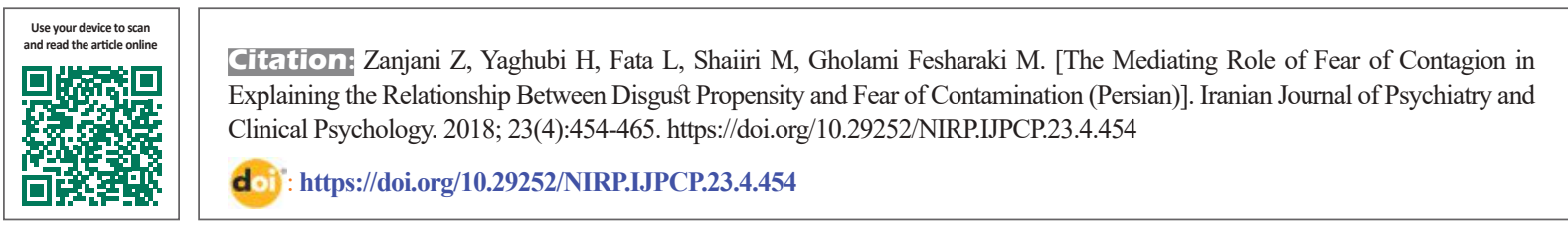

Received: 04 Jun. 2015

Accepted: 15 Mar. 2016

Key words:

Fear, Contamina-

tion, Student,

Disgust propensity,

Fear of contagion,

Mediating role

\section{A B STRACT}

Objectives The aim of the study was to investigate the relationship between disgust propensity, fear of contamination, and fear of contagion and also to determine the mediating role of fear of contagion in this regard.

Methods This is a correlation study. Study population were students of Shahed University of Tehran. The sample consisted of 391 students who were selected by convenience sampling method and completed looming contamination scale, disgust propensity and sensitivity scale and Padua scale. Data analyzed by SPSS 22 software. The correlation, hierarchical regression based on Baron and Kenny's method (1987), and Sobel's Test were used for analysis.

Results results showed that disgust propensity, fear of contagion ( $\beta=0.35, P<0.001)$, and fear of contamination $(\beta=0.41, P<0.001)$ are correlated. The relationship between fear of contagion and fear of contamination was also significant $(\beta=0.41, P<0.001)$. Regression analysis demonstrated that fear of contagion mediates the relationship between disgust propensity and fear of contamination $(\beta=0.26, P<0.001)$, which is also confirmed by Sobel's test $(Z=6.36, P<0.001)$.

Conclusion The fear of contagion is a mediator in the relationship between disgust propensity and fear of contamination. As a result, it seems that this structure is important in explaining the fear of contamination. Therefore if fear of contagion is considered in the evaluation and treatment of fear of contamination, The treartment effectiveness will be increased.

\section{Extended Abstract \\ most common OCD around the world [1] and also in Iran [2]. Recent research showed that individuals with F \\ 1. Introduction \\ ear of contamination is a dominant char- acteristic in Obsessive-Compulsive Dis- order (OCD), especially in the contam- ination-OCD (C-OCD) [1]. The C-OCD is one of the \\ C-OCD are more prone to experiencing disgust, and when faced with disgusting situations, they experience more disgust compared to others [3-5]. On the other hand, recent studies on disgust treatment revealed that this emotion is more resistant to habituation compared to fear and anxiety [6-10]. The fear of contagion has}

* Corresponding Author:

Hamid Yaghubi, PhD

Address: Department of Psychology, Faculty of Humanities, Shahed University, Tehran, Iran.

Tel: +98 (913) 2633275

E-mail: yaghubi@shahed.ac.ir 
been attributed to this resistance by some researchers [11-12]. It is said that people with disgust propensity believe in the contagion law; the law that Rozin and Fallon [13] have presented in the form of sympathetic magic beliefs. These beliefs refer to evaluations related to disgust that are based on the two thought processes of "contagion law" and "similarity law." According to the contagion law, once the objects come in contact they transfer some of their characteristics to each other, and therefore, affect each other in this way. This effect persists even after the physical contact is terminated [14].

Rozin et al. [14] found that most people tend to evaluate the potential of contamination of objects based on sympathetic magic beliefs. Sympathetic magic beliefs are stronger and more common in people with OCD because these people tend to stick to their thoughts, especially the disturbing obsessive-compulsive thoughts more than others [15]. Furthermore, Riskind [11] referred to a kind of cognitive vulnerability in anxiety disorders too, which appears in C-OCD in a way that these individuals perceive contamination as stimuli that spread close to themselves or stimuli with an increasing threat value [16]. These individuals overestimate the likelihood of spreading the contamination of contaminated object and transmitting it to neutral objects; in a way these people with C-OCD are said to have a looming vulnerability to contamination [17].

Although the fear of contagion associated with disgust and fear of contamination is of great interest and researchers have introduced it as a causative and mediating mechanism in this regard [18], no study has examined its role yet. In this scenario, the objectives of this research are twofold: 1 . To study the relationships between disgust propensity, fear of contagion, and fear of contamination; and 2. to examine the mediating role of fear of contagion in the relationship between disgust propensity and fear of contamination.

\section{Method}

The study sample included 391 students (285 female) from Shahed University of Tehran who were selected via available sampling. They completed the Looming Contamination Scale (LCS), the Padua Inventory (Contamination subscale), and the Disgust Propensity and Sensitivity Scale-Revised (DPSS-R). The average age of the participants was $21(\mathrm{SD}=4.02)$. Data were analyzed using SPSS 22 software. The Pearson correlation and the hierarchical regression in accordance with the Baron and Kenny [19] method and the Sobel Test were used.

\section{Results}

The results of the regression test revealed that there is a relationship between disgust propensity and fear of contagion $(\beta=0.35, \mathrm{P}<0.001)$. Moreover, a meaningful relationship was observed between fear of contagion and fear of contamination $(\beta=0.41, P<0.001)$. In this research, the Baron and Kenny method [19] was used to study the mediating role of fear of contagion. The results of the regression analyses (Table 1) showed that the four conditions of Baron and Kenny [19] are met, and the fear of contagion plays a mediating role in the relationship between disgust propensity and fear of contamination.

The reasons are as follows: 1 . There is a significant relationship between disgust propensity and fear of contamination $(\beta=0.0 .41, \mathrm{P}<0.001) ; 2$. There is a significant relationship between disgust propensity and fear of contagion ( $\beta=0.35, P<0.001) ; 3$. After controlling the effect of disgust propensity, there is a significant relationship between fear of contagion and fear of contamination $(\beta=0.41, \mathrm{P}<0.001)$; and 4. After controlling the fear of contagion, the relationship between disgust propensity and fear of contamination decreased from 0.41 to 0.26 (more than 0.1 ). The Sobel Test also demonstrated that the amount of change is significant $(\mathrm{Z}=6.36, \mathrm{P}<0.001)$. Therefore, it can be said that the fear

Table 1. The results of the regression analysis conducted to examine the mediating role of fear of contagion in the relationship between disgust propensity and fear of contamination

\begin{tabular}{|c|c|c|c|c|c|c|c|c|}
\hline $\begin{array}{c}\text { Regression } \\
\text { Models }\end{array}$ & Variables & $\begin{array}{c}\text { Independent } \\
\text { Variable }\end{array}$ & Total $\mathbf{R}^{2}$ & $\mathbf{F}$ & B & SE & Beta & $\begin{array}{r}\text { Sobel } \\
\text { Test }\end{array}$ \\
\hline 1 & Fear of contamination & Disgust propensity & 0.16 & 78.85 & 0.52 & 0.05 & 0.41 & 6.36 \\
\hline 2 & Fear of contagion & Disgust propensity & 0.12 & 55.39 & 0.88 & 0.11 & 0.35 & \\
\hline \multirow{2}{*}{3} & \multirow{2}{*}{ Fear of contamination } & Disgust propensity & \multirow{2}{*}{0.32} & \multirow{2}{*}{92.28} & 0.33 & 0.05 & 0.26 & \\
\hline & & Fear of contagion & & & 0.21 & 0.02 & 0.46 & \\
\hline
\end{tabular}



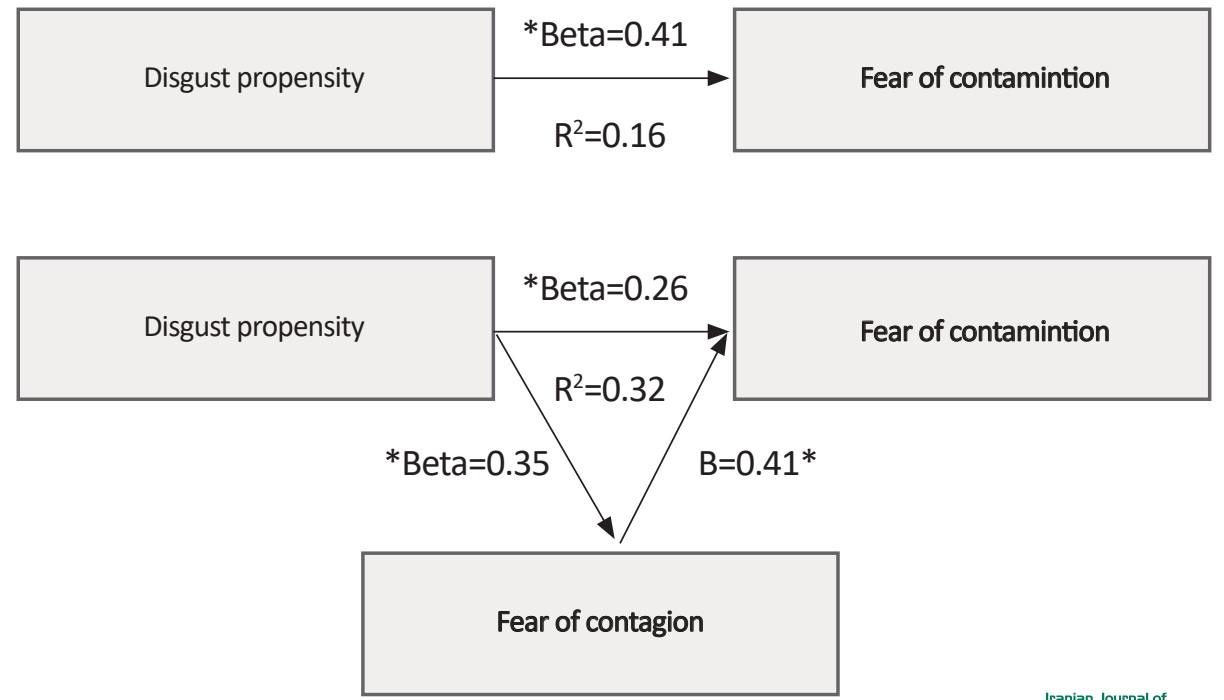

PSYCHIATRY AND CUINICAL PSYCHOLOGY

Figure 1. Fear of contagion mediated the relationship between disgust propensity and fear of contamination

of contagion relatively mediates the relationship between disgust propensity and fear of contamination.

\section{Discussion}

The first aim was to study the relationships between fear of contagion, fear of contamination, and disgust propensity. The results indicated that there was a significant relationship between disgust propensity, fear of contagion ( $\mathrm{r}=0.35$ ) and fear of contamination $(\mathrm{r}=0.41)$. Also, there was a discernible relationship between fear of contagion and fear of contamination $(r=0.51)$. These results are consistent with Olatunji et al. [3], Broadrick [4], Riskind et al. [16], and Tolin et al. [17].

It is said that disgust act as an adaptive and protective system against diseases [20]. This emotion mostly protects a person against diseases and leads to a person's avoidance of contaminated stimuli [21]. This avoidance makes it easier to understand the relationship between disgust and the fear of contamination. The findings of this research also revealed that the individuals with disgust propensity were more concerned and afraid about contamination. Furthermore, according to McKay [15], it seems that those who are more afraid of contagion tend to suspect that an object may be contaminated more than others due to this belief; as a result, they become worried about being contaminated by contact with this object.

Another goal of this study was to examine the mediating role of fear of contagion in the relationship between disgust propensity and fear of contamination (Figure 1). As predicted, the findings of this research supported the mediating role of this construct. This finding suggested the importance of fear of contagion in explaining the fear of contamination. The results were in line with previous studies [18] as the results showed that the individuals with disgust propensity believed more in the fear of contagion. According to Riskind et al. [16], this belief works similar to the danger schema, which makes a person sensitive to the signs of contamination and danger, and causes anxiety [11].

In summary, the fear of contagion is a mediating variable in the relationship between disgust propensity and fear of contamination. Our findings suggested that even though disgust propensity is an important component of fear of contamination, a person with no fear of contagion experiences less fear of contamination. Consequently, it appears that this construct is important in explaining the fear of contamination. Finally, it is noteworthy to mention that a treatment will be more effective if it considers fear of contagion when evaluating and treating fear of contamination.

\section{Ethical Considerations}

\section{Compliance With Ethical Guideline}

After completing the informed consent form, participants completed the questionnaires.

\section{Funding}

This paper was extracted from the $\mathrm{PhD}$ dissertation of the first author in the Department of Clinical Psychology, Faculty of Medicine, Kashan University of Medical Sciences.

\section{Conflict of Interest}

The authors declare no conflict of interest. 


\title{
نقش واسطهاى ترس از سرايت در تبيين رابطه بين تحرايش به تجربه جهندش و ترس از آلودتى
}

\author{
زهرا زنجانى'? "حميد يعقوبى" لادن فتى"، محمدرضا شعيرى"، محمد غلامى فشاركى"

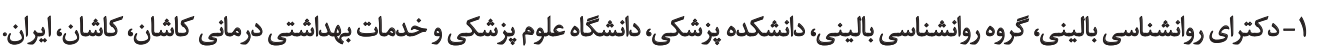

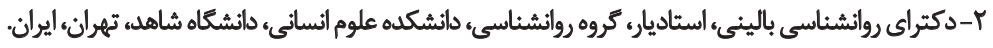

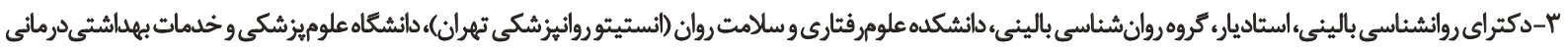

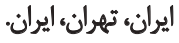

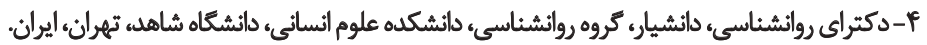

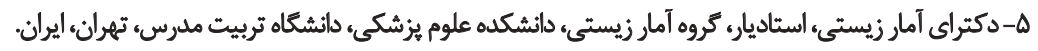

\begin{abstract}
حكبد

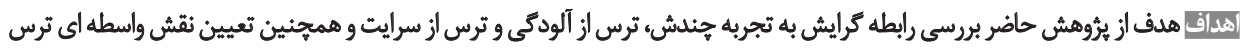

ازاز سرايت در اين رابطه بود حان.

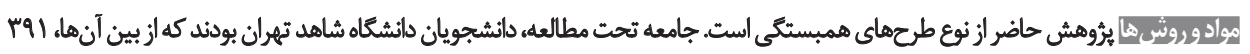

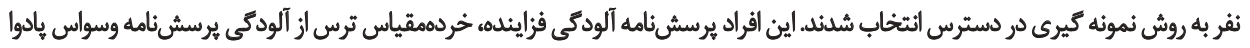

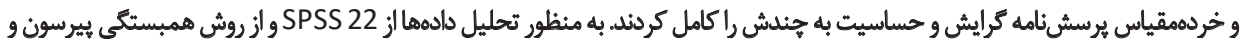

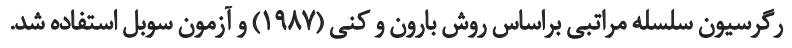

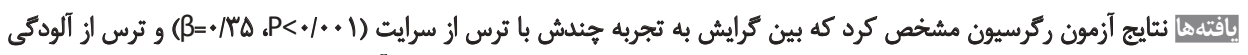

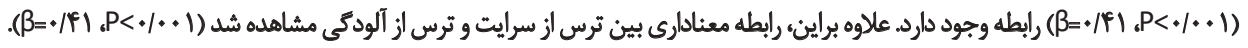

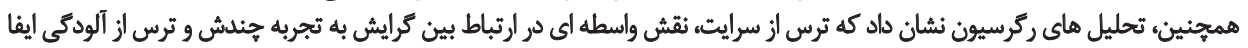

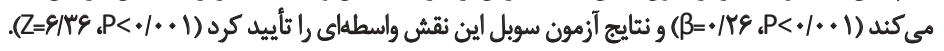

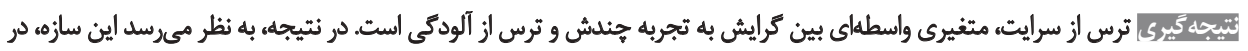

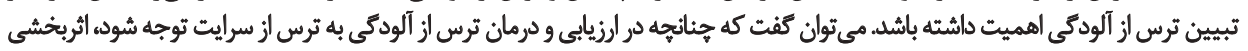
درمان افزايش مي اليد.
\end{abstract}

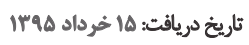

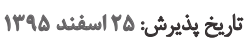

كليدوازهذها:

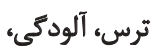

دانشجو،كرايش به تجربه جندش، ترس ازٔ سرايت، نقش واسطهاى

$$
\text { از كل جمعيت) را مبتلا مى كند [ب]. }
$$

مقدمه

يكى از شايعترين انواع اختلال وسواس در جهان [1 إنى و و ايران

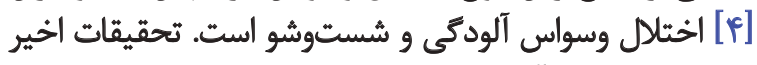

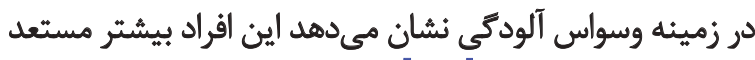

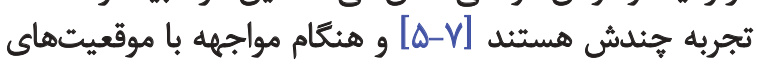

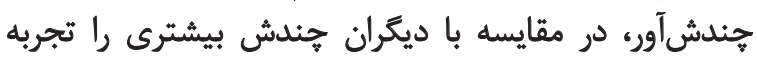

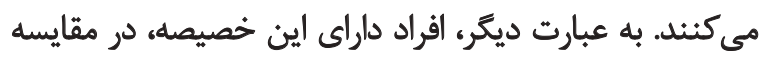

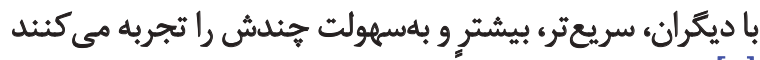

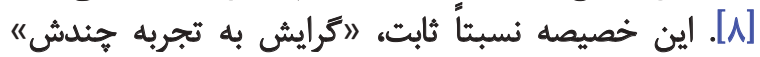

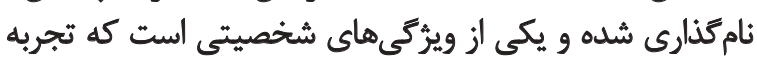

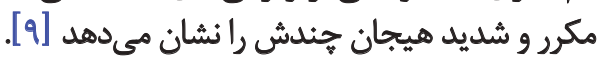

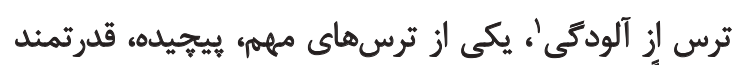

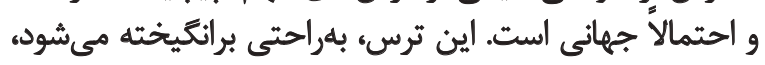

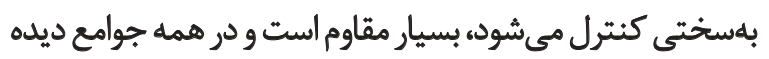

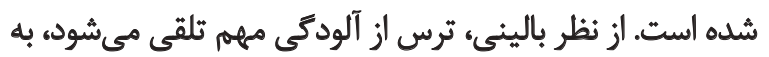

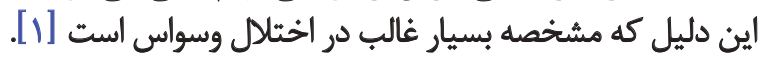

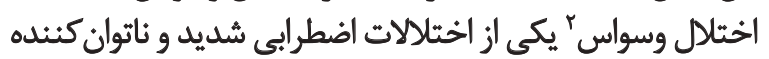

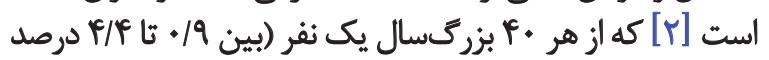

1. Fear of contamination

2. Obsessive Compulsive Disorder (OCD) 


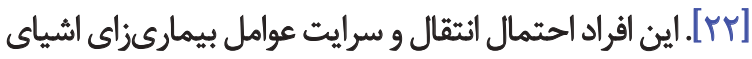

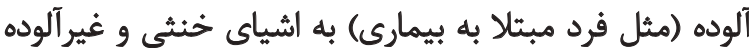

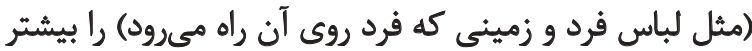

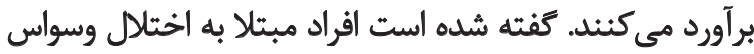

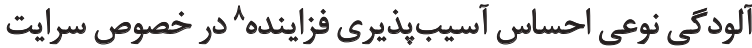

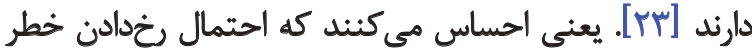

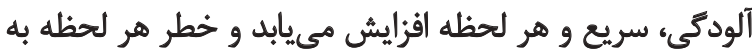

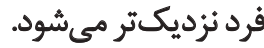

درباره سبك شناختى فزاينده (LCS)'، كفته شده است كه

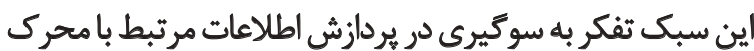

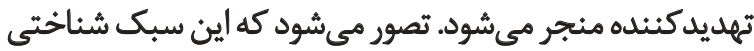

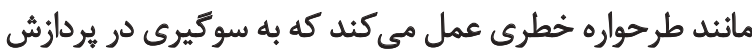

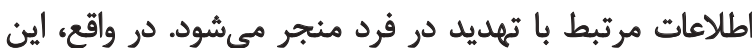

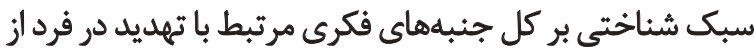

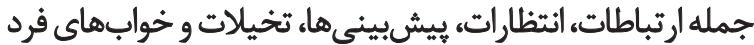

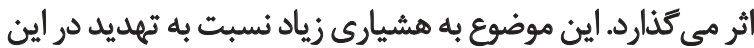

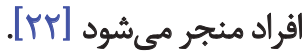

دانشجويان با علائم وسواسى، هنكام تصور صحنههاي مرتبط

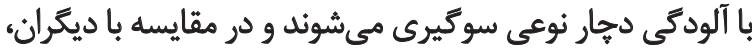

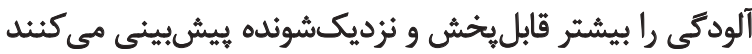

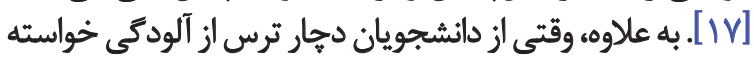

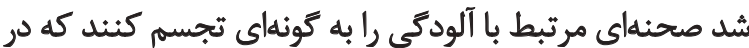

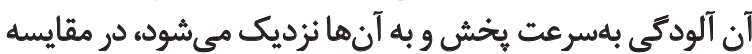

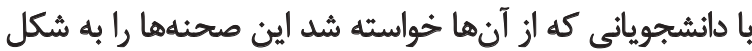

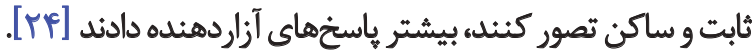

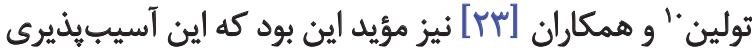

$$
\text { در ثرس از آلودنى نقش دارد. }
$$

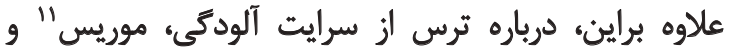

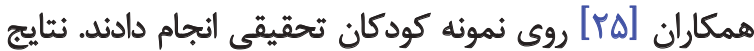

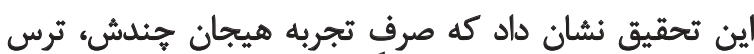

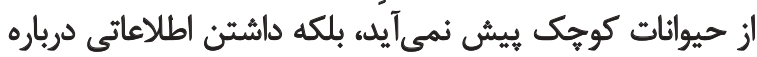

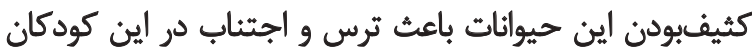

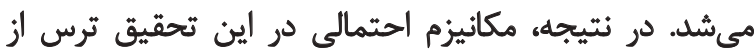

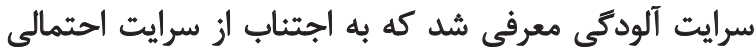

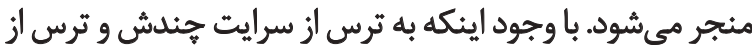

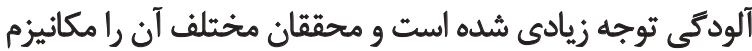

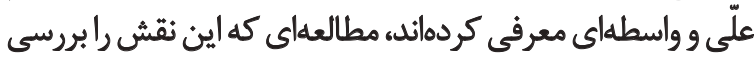

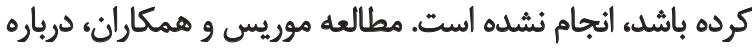

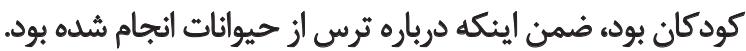

8. Looming vulnerability

9. Looming Cognitive Style (LCS)

10. Tolin

11. Muris

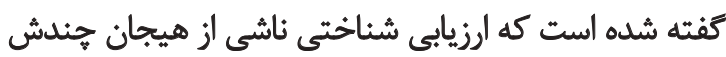
ممكن است باعث بيش تخمين نتايج تماس باس با آلودكى شئ شود

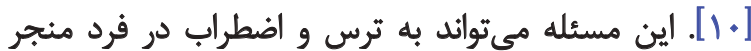

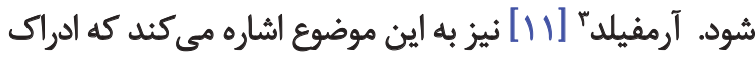

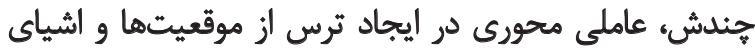

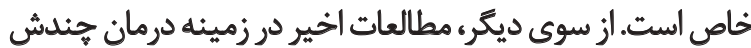

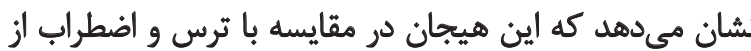

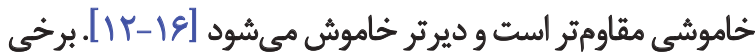

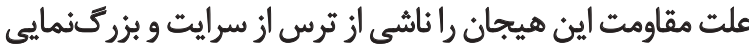

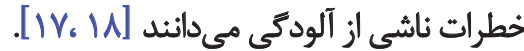

در توضيح اين موضوع تفته شده است افراد داراى اين هيجان

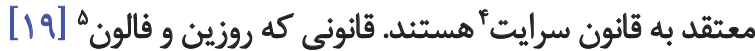

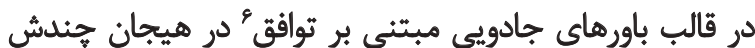

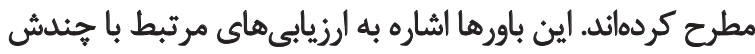
دارد كه بر اساس دو فرايند فكرى "اقانون سرايت" و و القانون

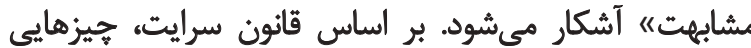

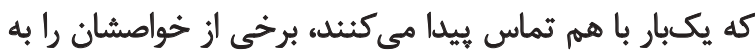

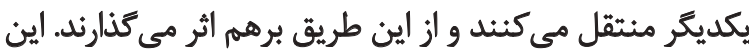

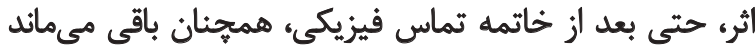

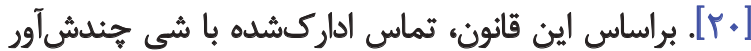

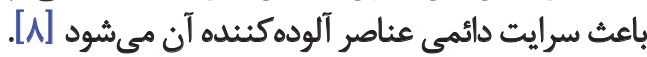
دومين فرايند فكرى، "اقانون مشابهت ") است و به اين موضوع

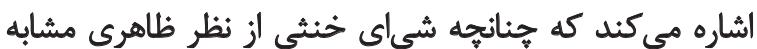

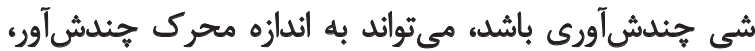

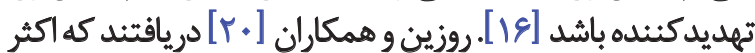

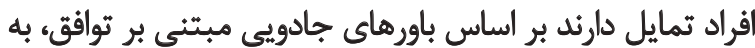

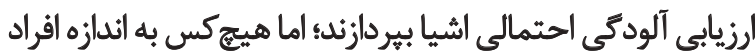

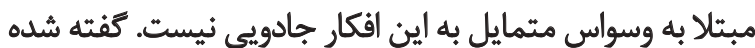

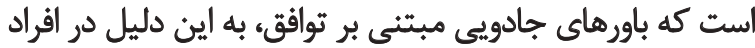

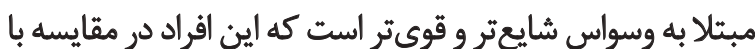

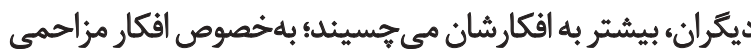

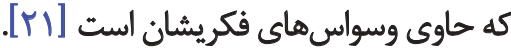
علاوه بر اين، ريسكيند [IV] علت اين موضوع را وجود نوعى إني

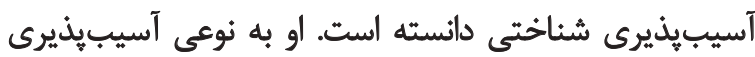

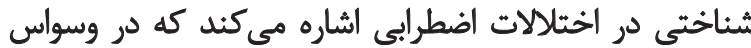

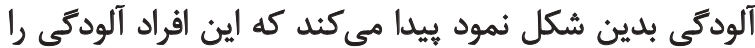

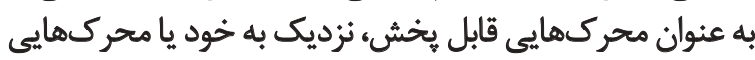

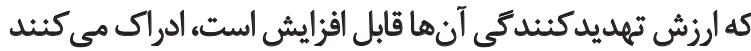
3. Armfield
4. Contagion law
5. Fallon
6. Sympathetic magic beliefs
7. Low of similarity 
شركت و همكارى در يرؤهش به آنها هديهاى داده شد.

ابزارها

\section{خردهمقياس آلودكى يرسش}

ساناوى در سال 1911 يرسشنامه يادوا را تهيه كرد كه شامل

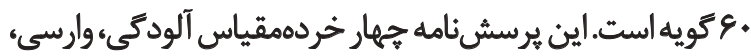

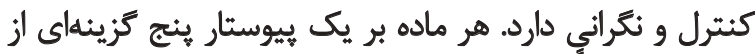

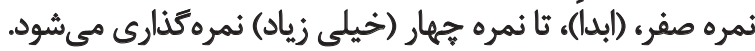

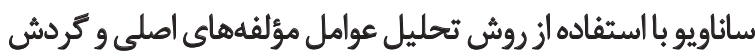

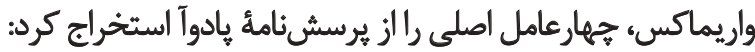

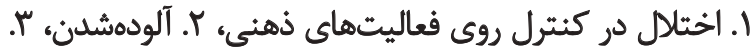

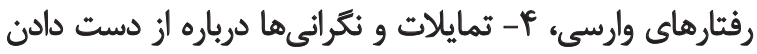

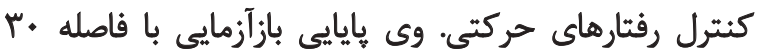

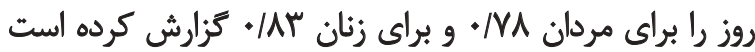

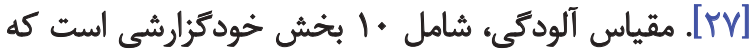

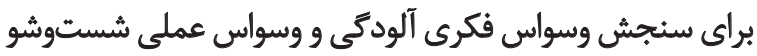

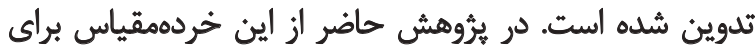
سنجش ترس از آلودگى استفاده شد.

كودرزى و فيروزآبادى [rV] درباره ساختار عاملى و وإياييى

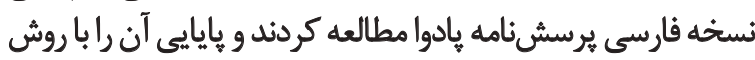

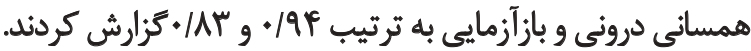

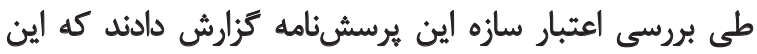

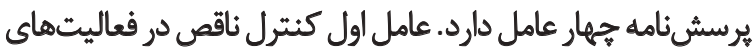

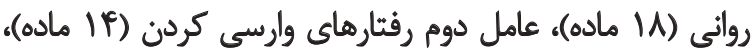

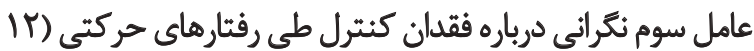

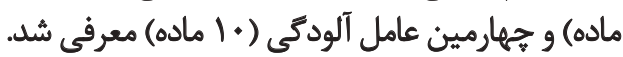

خرده مقياس ترايش به تجربه جندش مقياس حساسيت

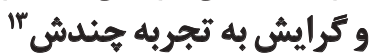

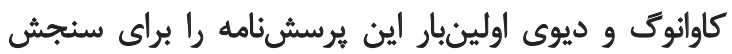

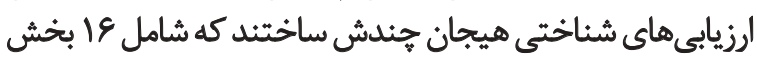

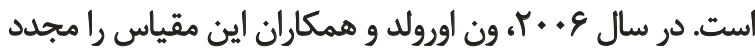

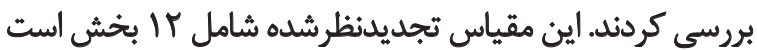

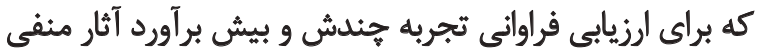

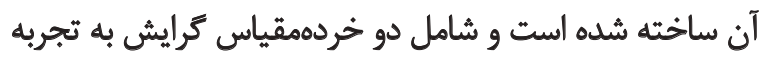

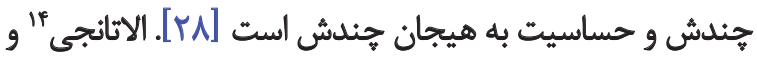

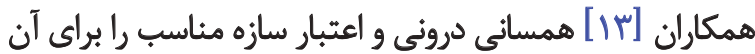

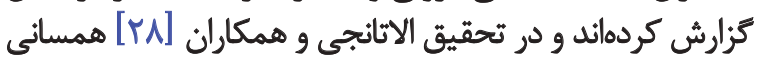

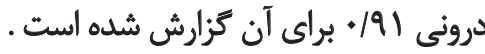

12. Padua Inventory (PI)

13. Disgust Propensity and Sensitivity Scale (DPSS) 14. Olatunji

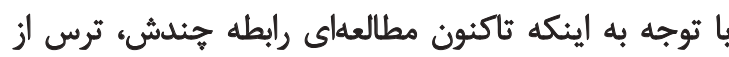

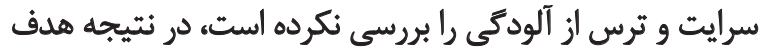

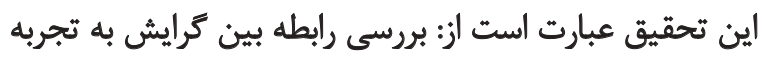

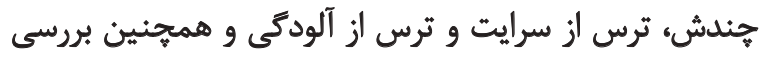

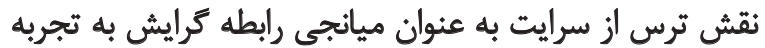

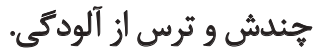

و

مطالعه حاضر از نوع يُروهش هاى همبستيكى است. جامعه

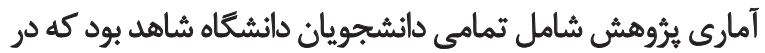

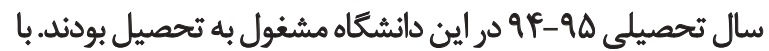

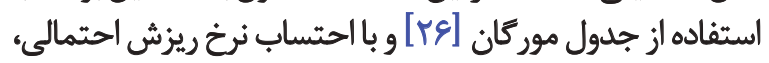

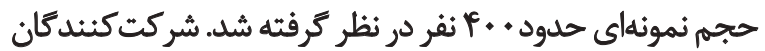

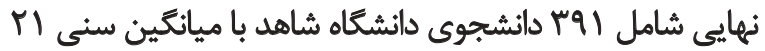

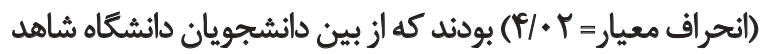

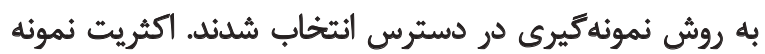

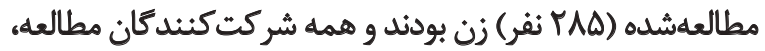

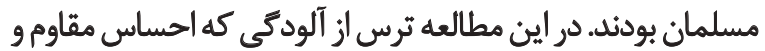

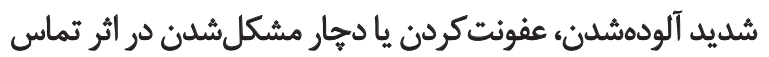

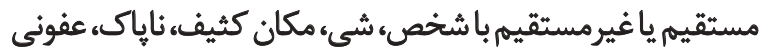

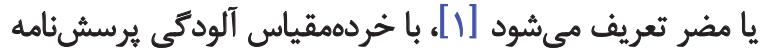
وسواس يادوا سنجيده شد.

متغير ترايش به تجربه جندش (كرايش عمومى به تجربه مبر مكرر

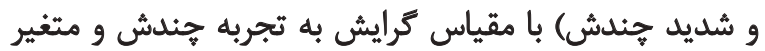

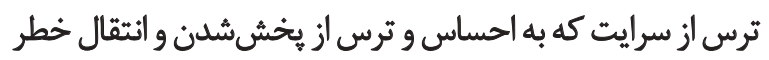

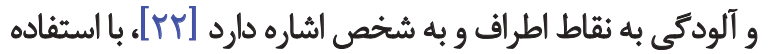

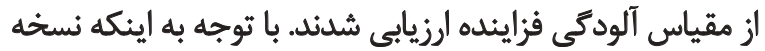

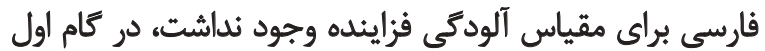

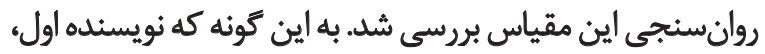

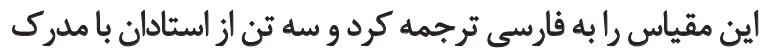

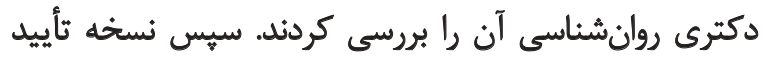

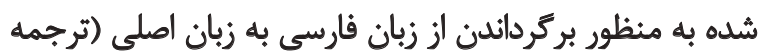

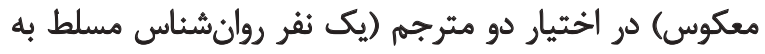

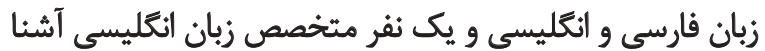

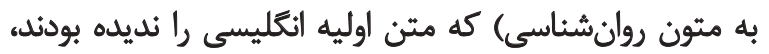

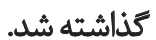

در ادامه، اين نسخه با نسخه اصلى مقايسه شد و درباره موارد

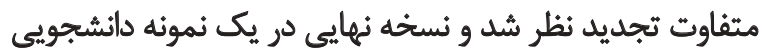

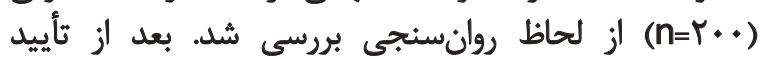

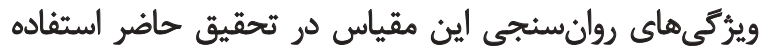

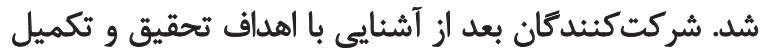

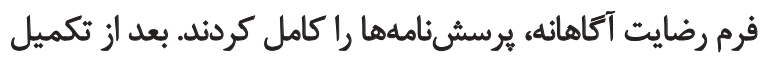

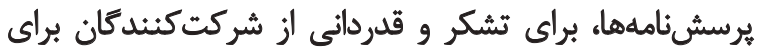




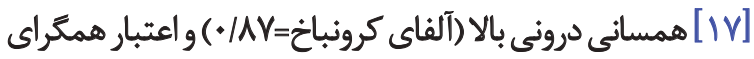

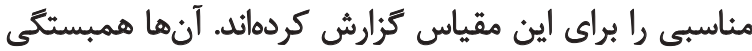

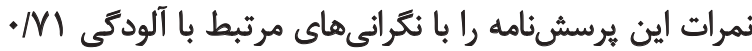

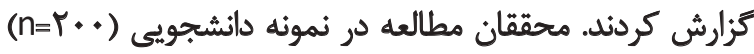

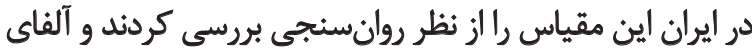

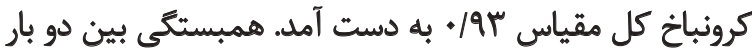

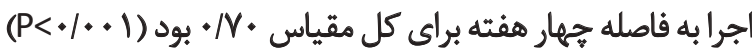

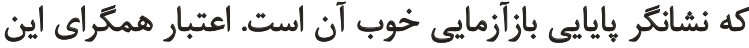

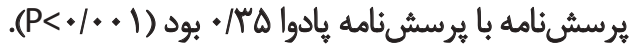

$$
\text { تماليل آمارى }
$$

تحليل دادهها با استفاده از نرمافزار

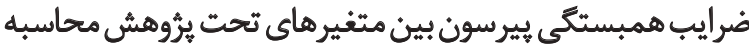

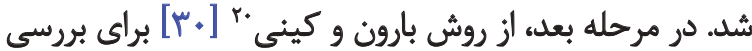

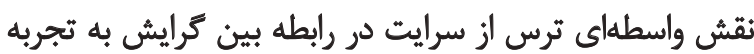

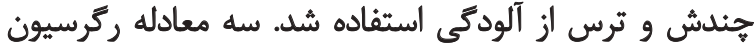

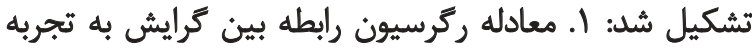

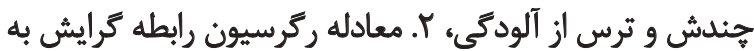

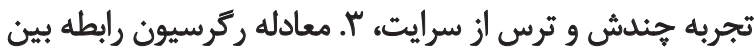

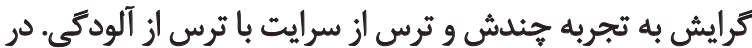

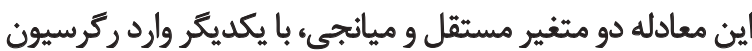
مىشوند. در نهايت، براى آزمون معنادارى اثر واسطهائ ازئ آزمون

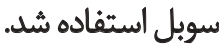

ياقتههها

جدول شماره 1 نتايج همبستگى بيرسون بين متغيرهاى

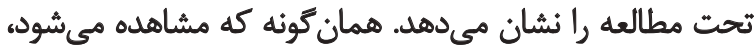

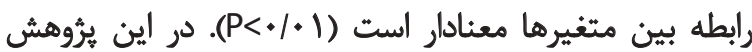

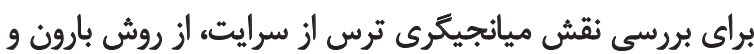

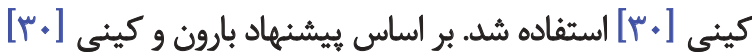

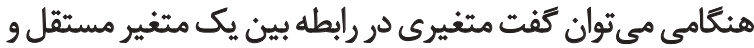

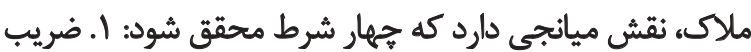

20. Baron \& Kenny

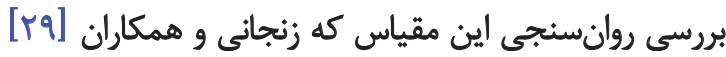

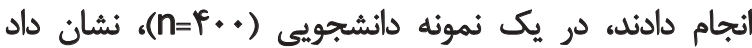

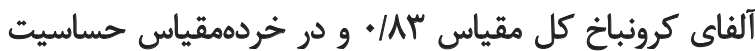

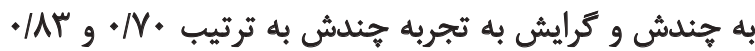

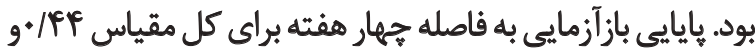

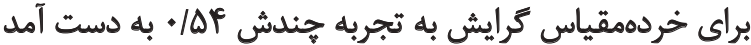

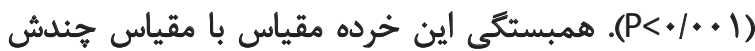

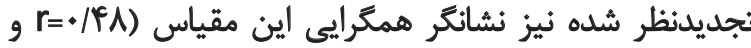

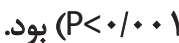

\section{مقياس آلوديّى فزايندهها}

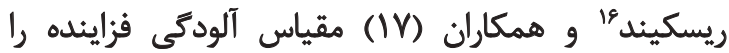

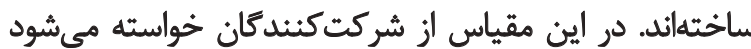

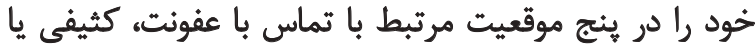

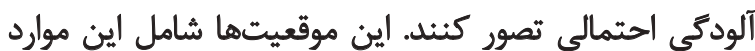

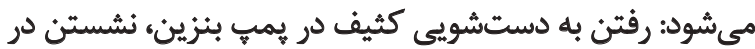

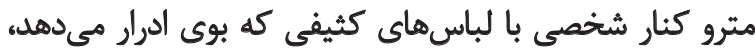

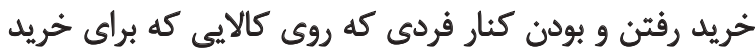

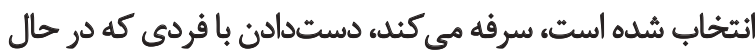

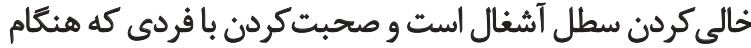
صحبت، روى زمين تف مي اندازد.

سيس، از شركتكنئده خواسته مىشود بعد از تصور خود

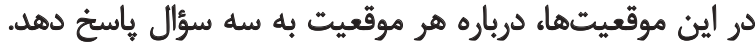

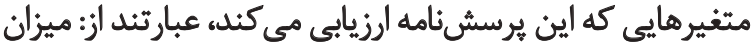

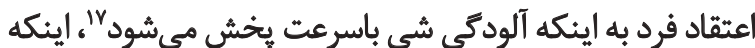

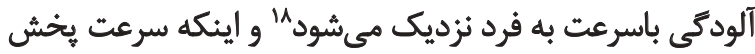

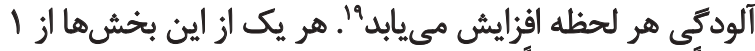

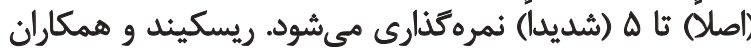

15. Looming Cognitive Style (LCS)

16. Riskind

17. Speed of spread

18. Rapidity of approach

19. Acceleration

جدول ا. نتايج آزمون همبستكى بيرسون و ميانكين و انحراف معيار متغيرهاى تحت مطالعه

\begin{tabular}{|c|c|c|c|}
\hline ترس از سرايت & مرايش بيه تجربه هندش & توس از آلودكى & هتغيرها \\
\hline $.101^{\circ}$ &.$|4|^{*}$ & - & ترس أز ألودتى \\
\hline.$/ M \Delta^{*}$ & - &.$|x|^{*}$ & كرايش به تجربه هندش \\
\hline- &.$/ N \Delta^{\circ}$ & $. \mid \Delta)^{\circ}$ & ترس از سرايت \\
\hline$P f /(M r / U)$ & $\Gamma / T r(\Delta / 1 \Delta)$ & $1 . / T \Delta(\% / \Delta A)$ & ميانكين (انحراف معيار) \\
\hline
\end{tabular}


جدول r. تنايج تحليل ركرسيون به منظور آزمون نقش ميائجيكرى ترس از سرايت در رابطه بين كرايش به تجربه جندش و ترس از آلودىى

\begin{tabular}{|c|c|c|c|c|c|c|c|c|}
\hline Sobel Test & Beta & SE & B & $F$ & Total $\mathbf{R}^{r}$ & مثغير هاى مسثقل & مثغير ملاكى & رعادله \\
\hline \multirow{3}{*}{ g/Ne } &.$M I^{*}$ & $.1 \cdot 0$ & . $/ A Y$ & VNAD & .118 & كرايش به تجريه جندش & ترس از ألودكى & معادله اول \\
\hline &.$/ r \omega^{*}$ & .111 & $\cdot / M$ & $\Delta \Delta / r q$ &.$/ M$ & كرايش به تجربه جندش & ترس ازٔ سرايت & معادله دوم \\
\hline & $\begin{array}{l}. / K E * \\
.|K|^{*}\end{array}$ & $\begin{array}{l}.1+0 \\
.1+r\end{array}$ &.$/ M$ & QT/TA & . & كرايش بله تخربه جندش ترس از & ترس از آلود5ى & معادله سوم \\
\hline
\end{tabular}

كفت متغير مدنظر در رابطه بين متغير مستقل و وابسته نقش مانش

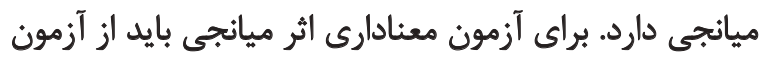

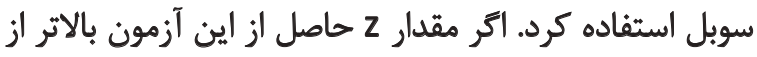
1/98 باشد، مقدار اثر متغير ميانجى معنادار است آحل

بر اين اساس نتايج تحليل هاى ركرسيون به منظور وارسى نقش مئش

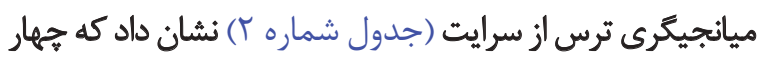

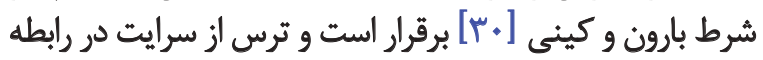

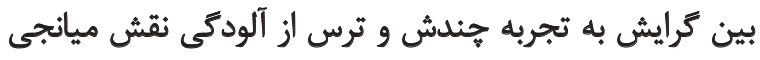

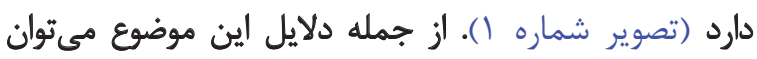

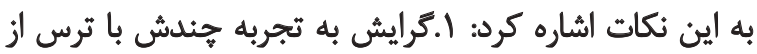

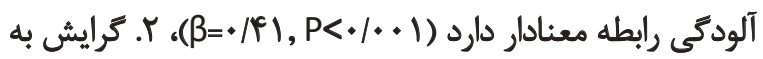

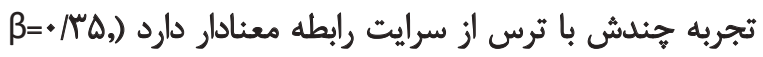

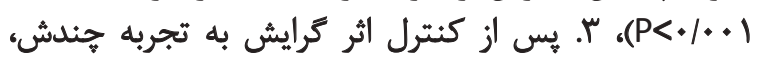

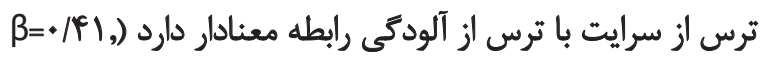

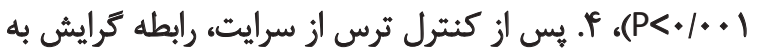

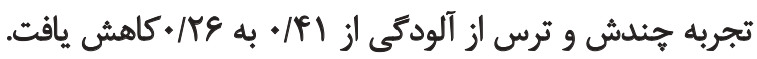

استاندارد (بثا) بين متغير مستقل و متغير وابسته معنادار باشد

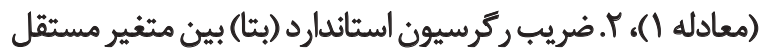

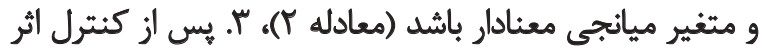

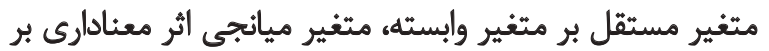

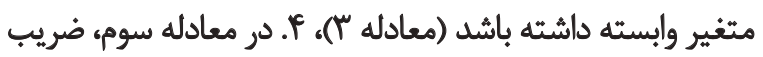

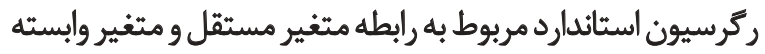

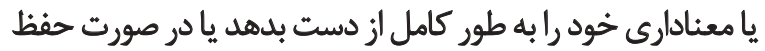

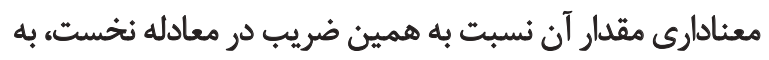
نحو معنادارى كاهش يابد (بيشنهادها در حد || /.). در صورتى كه ضريب ركرسيون استاندارد مربوط به رابطه

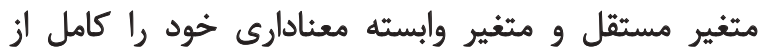

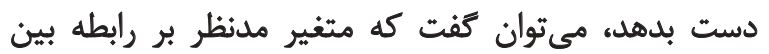

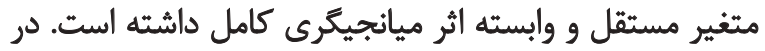

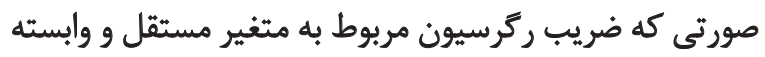

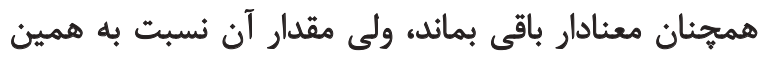

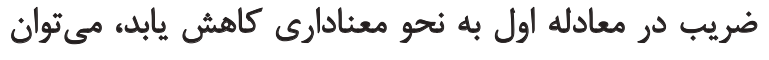
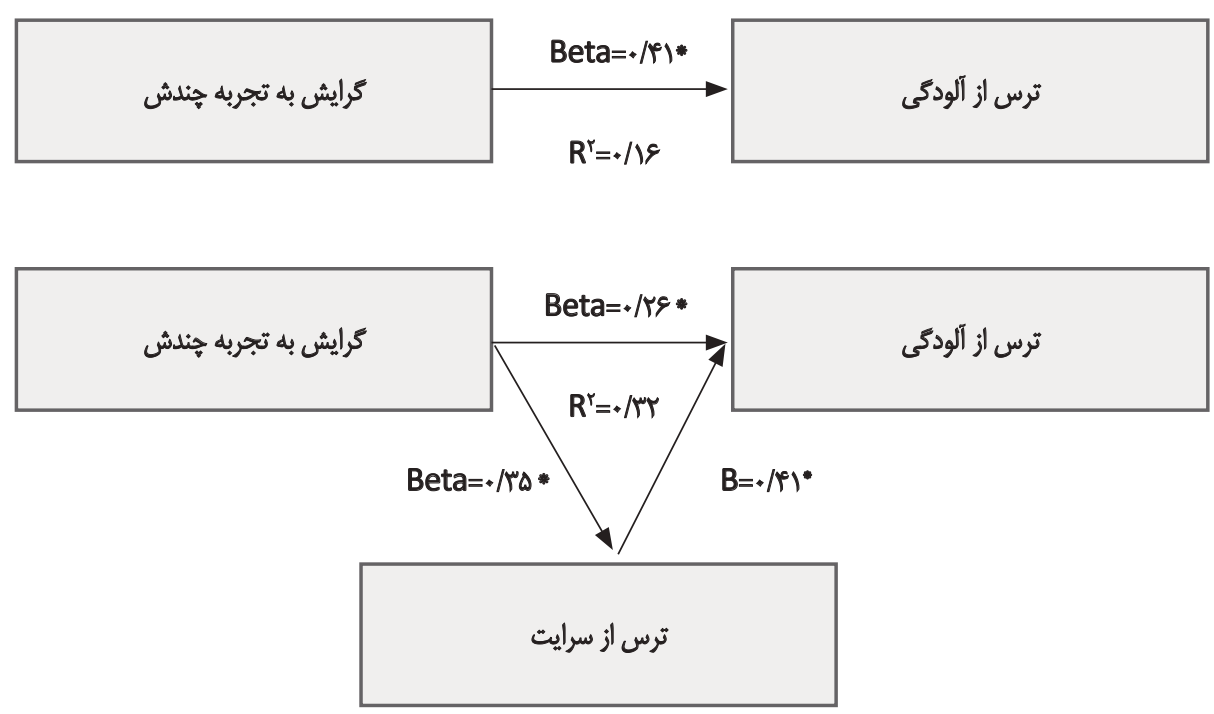

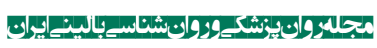

تصوير ا. ترس از سرايت، به طور نسبى رابطه كرايش به تجربه جندش و ترس از آلودكى را ميانجيكرى مى كند. $\bullet P<\cdot 1 \cdot+1$ Sobel's Test: $Z=\$, \pi \%, P=.1 . .1$ 


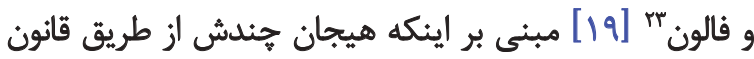

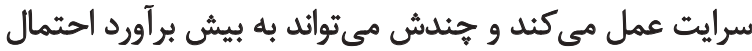

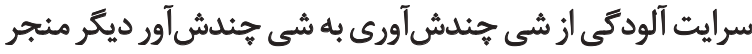
شود، فراهم كرد. بر طبق قانون سرائ سرايت در نظريه

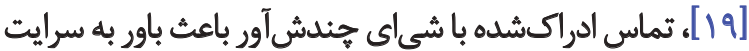

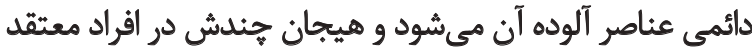

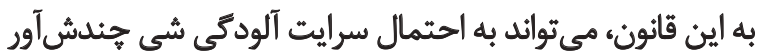

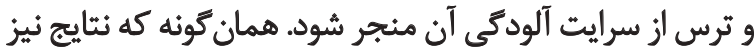

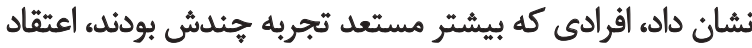

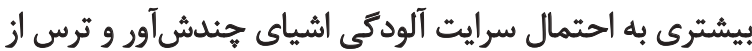

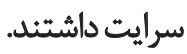

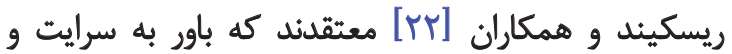

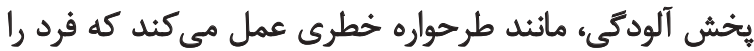

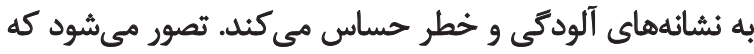

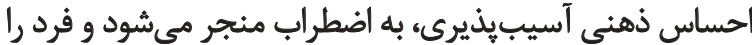

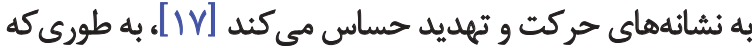

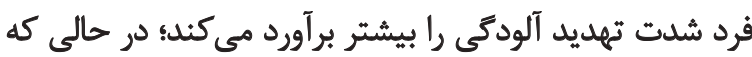

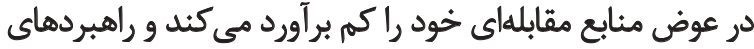

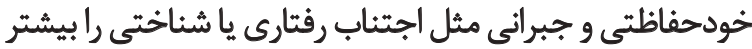

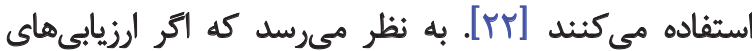

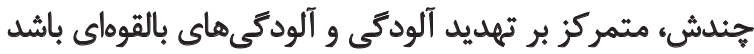

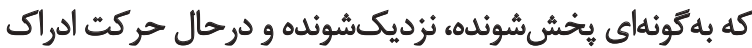

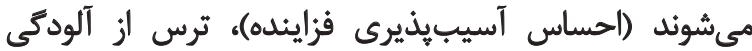

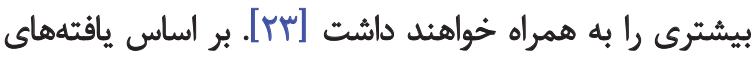

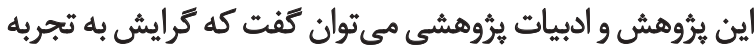

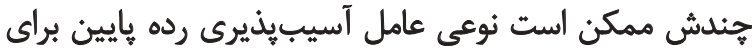

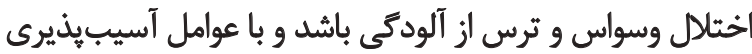
رده بالاتر مثل ترس از سرايت تقويت شود.

با توجه به اينكه مطالعه حاضر، مطالعهاى مقطعى و از از إز

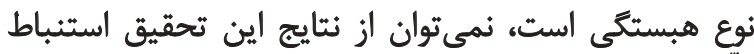

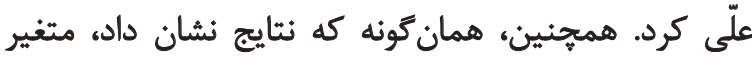

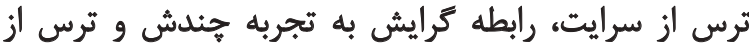

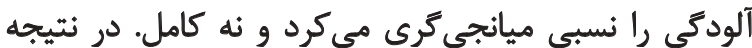

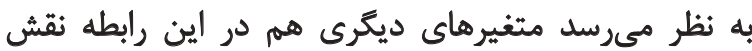

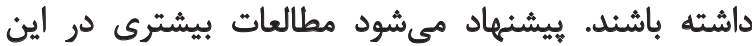

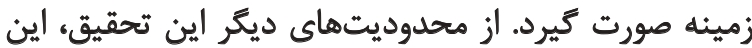

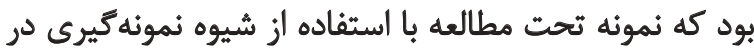

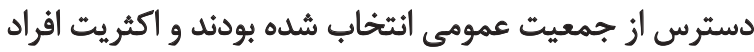

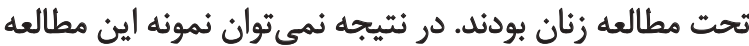

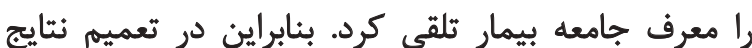

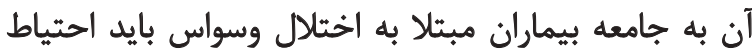

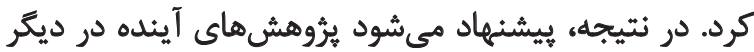

كرحها معنادارى حُود را حفظ كرده است، اين كاهش بيشتر از

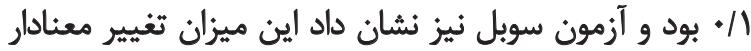

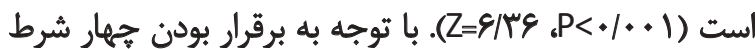

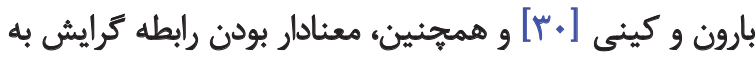

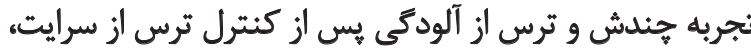

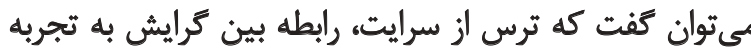
جندش و ترس از آلودكى را به صورت نسبى ميانجيكرى مى كند.

بحث

هدف اول اين تحقيق بررسى رابطه بين ترس از سرايت،

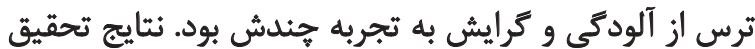

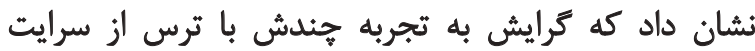
همجاته

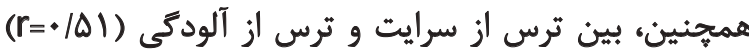

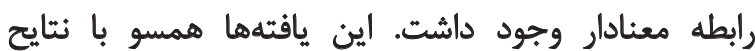

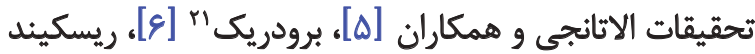

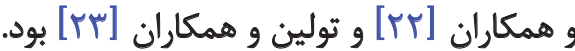

كفته شده است كه هيجان جندش مائند سيستم انطباقى و

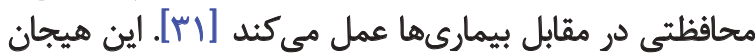

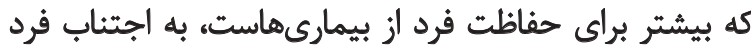

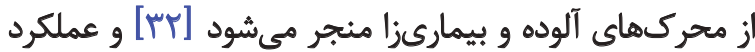

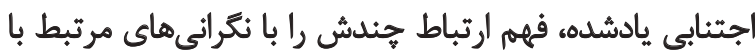

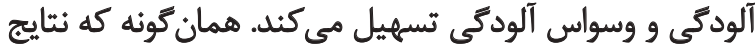

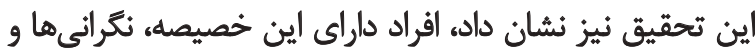

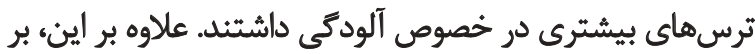

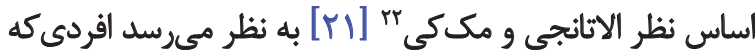

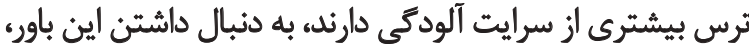

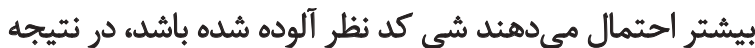

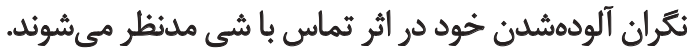

هدف ديكر اين مطالعه بررسى نقش واسطهاي ترس از

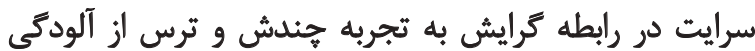

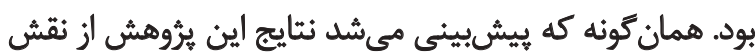

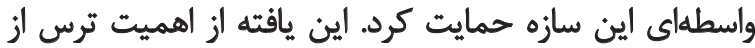

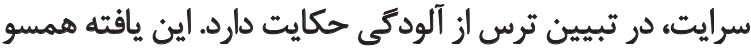

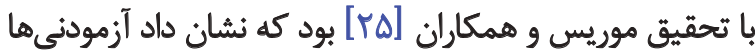

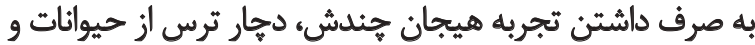

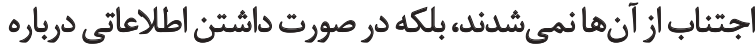

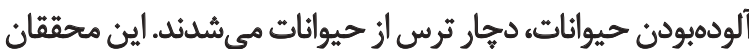

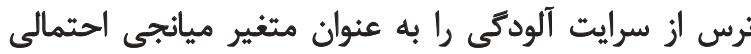

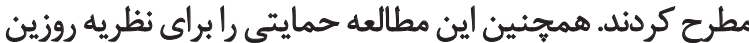

21.Broderick

22. Olatunji \& McKay 
جمعيتها، به ويرٔه جمعيت بالينى صورت كيرد.

با وجود اين محدوديتها، نتايج مطالعه حاضر كاربردهاى براي

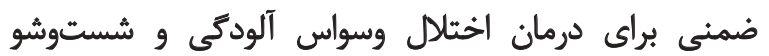

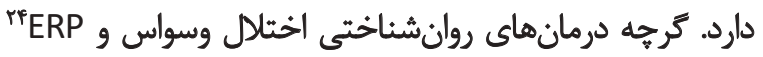

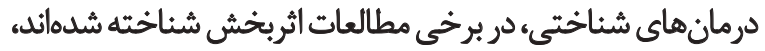

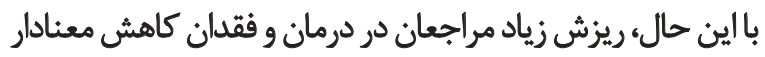

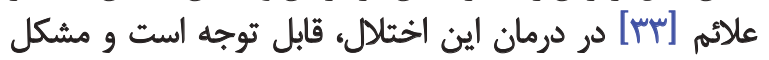

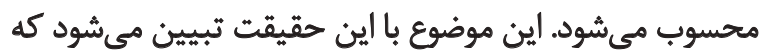

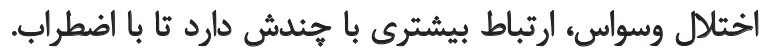

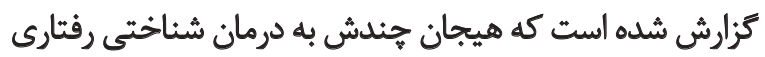

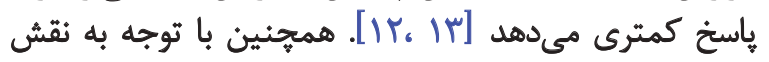

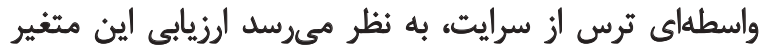

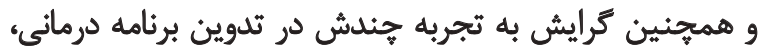

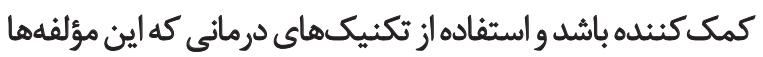
را هدف قرار دهد، اثربخشى درمان را افزايش دهدي.

$$
\text { نتيجليرى }
$$

بر اساس نتايج اين بثوهش مي توان كفت كه كرجه كرايش به

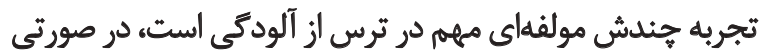

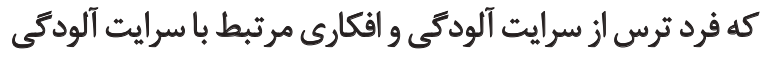

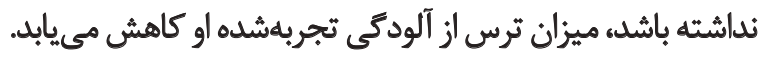

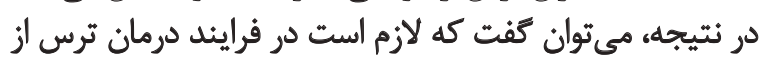

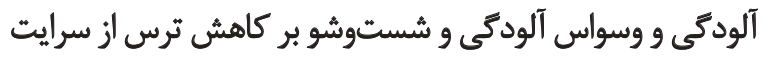

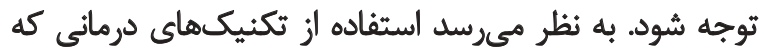

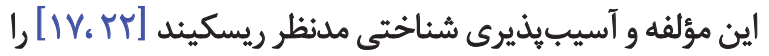

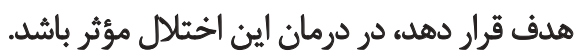

$$
\text { سياسكَّزارى }
$$

در پايان لازم است از تمامى دانشجويان و استادان محترم

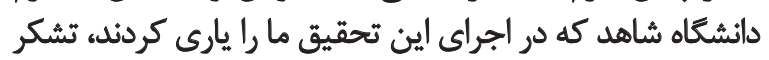

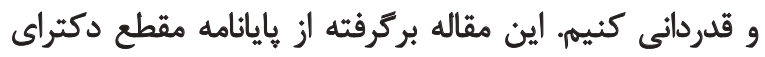

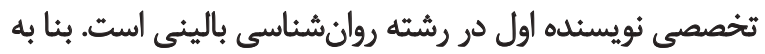

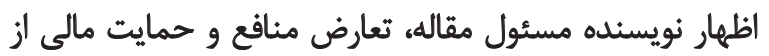

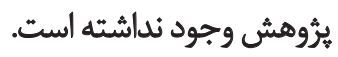




\section{References}

[1] Rachman S. Fear of contamination. Behaviour Research and Therapy. 2004; 42(11):1227-55. doi: 10.1016/j.brat.2003.10.009

[2] Bobes J, González MP, Bascarán MT, Arango C, Sáiz PA, Bousoño M. Quality of life and disability in patients with obsessivecompulsive disorder. European Psychiatry. 2001; 16(4):239-45. doi: 10.1016/s0924-9338(01)00571-5

[3] Merikangas KR. Anxiety disorders: Epidemiology. In: Sadock BJ Sadock VA, editors. Kaplan \& Sadock's Comprehensive Textbook of Psychiatry. Philadelphia: Lippincottt William \& Wilkins; 2005.

[4] Dadfar M, Bolhari J, Dadfar K, Bayanzadeh SA. [Prevalence of the obsessive-compulsive disorder symptoms (Persian)]. Iranian Journal of Psychiatry and Clinical Psychology . 2001; 7(1-2):27-33.

[5] Olatunji BO, Tart CD, Ciesielski BG, McGrath PB, Smits JAJ. Specificity of disgust vulnerability in the distinction and treatment of OCD. Journal of Psychiatric Research. 2011; 45(9):1236-42. doi: 10.1016/j.jpsychires.2011.01.018

[6] Broderick J. Disgust and contamination fear based obsessivecompulsive disorder: an investigation [PhD dissertation]. Kensington: University of New South Wales; 2012.

[7] Goetz AR, Lee HJ, Cougle JR, Turkel JE. Disgust propensity and sensitivity: Differential relationships with obsessive-compulsive symptoms and behavioral approach task performance. Journal of Obsessive-Compulsive and Related Disorders. 2013; 2(4): 412-9. doi: 10.1016/j.jocrd.2013.07.006

[8] Cisler JM, Olatunji BO, Lohr JM, Williams NL. Attentional bias differences between fear and disgust: Implications for the role of disgust in disgust-related anxiety disorders. Cognition \& Emotion. 2009; 23(4):675-87. doi: 10.1080/02699930802051599

[9] Van Overveld WJM, De Jong PJ, Peters ML, Cavanagh K, Davey GCL. Disgust propensity and disgust sensitivity: Separate constructs that are differentially related to specific fears. Personality and Individual Differences. 2006; 41(7):1241-52. doi: 10.1016/j. paid.2006.04.021

[10] Deacon B, Olatunji BO. Specificity of disgust sensitivity in the prediction of behavioral avoidance in contamination fear. Behaviour Research and Therapy. 2007; 45(9):2110-20. doi: 10.1016/j. brat.2007.03.008

[11] Armfield JM. Cognitive vulnerability: A model of the etiology of fear. Clinical Psychology Review. 2006; 26(6):746-68. doi: 10.1016/j.cpr.2006.03.007

[12] Olatunji BO, Forsyth JP, Cherian A. Evaluative differential conditioning of disgust: A sticky form of relational learning that is resistant to extinction. Journal of Anxiety Disorders. 2007; 21(6):82034. doi: 10.1016/j.janxdis.2006.11.004

[13] Olatunji BO, Smits JAJ, Connolly K, Willems J, Lohr JM. Examination of the decline in fear and disgust during exposure to threat-relevant stimuli in blood-injection-injury phobia. Journal of Anxiety Disorders. 2007; 21(3):445-455. doi: 10.1016/j. janxdis.2006.05.001

[14] Smits JAJ, Telch MJ, Randall PK. An examination of the decline in fear and disgust during exposure-based treatment. Behaviour Research and Therapy. 2002; 40(11):1243-53. doi: 10.1016/s00057967(01)00094-8
[15] McKay D. Treating disgust reactions in contamination-based obsessive-compulsive disorder. Journal of Behavior Therapy and Experimental Psychiatry. 2006; 37(1):53-9. doi: 10.1016/j. jbtep.2005.09.005

[16] Olatunji BO, Wolitzky-Taylor KB, Willems J, Lohr JM, Armstrong T. Differential habituation of fear and disgust during repeated exposure to threat-relevant stimuli in contaminationbased OCD: An analogue study. Journal of Anxiety Disorders. 2009; 23(1):118-23. doi: 10.1016/j.janxdis.2008.04.006

[17] Riskind JH, Abreu K, Strauss M, Holt R. Looming vulnerability to spreading contamination in subclinical OCD. Behaviour Research and Therapy. 1997; 35(5):405-14. doi: 10.1016/s00057967(96)00113-1

[18] Olatunji BO, Sawchuk CN, Lohr JM, De Jong PJ. Disgust domains in the prediction of contamination fear. Behaviour Research and Therapy. 2004; 42(1):93-104. doi: 10.1016/s00057967(03)00102-5

[19] Rozin P, Fallon AE. A perspective on disgust. Psychological Review. 1987; 94(1):23-41. doi: 10.1037//0033-295x.94.1.23

[20] Rozin P, Millman L, Nemeroff C. Operation of the laws of sympathetic magic in disgust and other domains. Journal of Personality and Social Psychology. 1986; 50(4):703-12. doi: 10.1037//0022 3514.50.4.703

[21] Olatunji BO, McKay D. Disgust and its disorders: Theory, assessment, and treatment implications. Washington, D.C.: American Psychological Association; 2009

[22] Riskind JH, Williams NL, Joiner TE. The looming cognitive style: A cognitive vulnerability for anxiety disorders. Journal of Social and Clinical Psychology. 2006; 25(7):779-801. doi: 10.1521/ jscp.2006.25.7.779

[23] Tolin DF, Worhunsky P, Maltby N. Sympathetic magic in contamination-related OCD. Journal of Behavior Therapy and Experimental Psychiatry. 2004; 35(2):193-205. doi: 10.1016/j. jbtep.2004.04.009

[24] Williams NL, Connolly KM, Cisler JM, Elwood LS, Williams JL, Lohr JM. Disgust: A cognitive approach. In: Olatunji BO, McKay D, editor. Disgust and Its Disorders: Theory, Assessment, and Treatment Implications. Washington, D.C.: American Psychological Association; 2009.

[25] Muris P, Mayer B, Huijding J, Konings T. A dirty animal is a scary animal! Effects of disgust-related information on fear beliefs in children. Behaviour Research and Therapy. 2008; 46(1):137-44. doi: 10.1016/j.brat.2007.09.005

[26] Krejcie RV, Morgan DW. Determining sample size for research activities. Educational and Psychological Measurement. 1970; 30(3):607-10. doi: 10.1177/001316447003000308

[27] Goodarzi MA, Firoozabadi A. Reliability and validity of the Padua Inventory in an Iranian population. Behaviour Research and Therapy. 2005; 43(1):43-54. doi: 10.1016/j.brat.2003.11.004

[28] Olatunji BO, Ebesutani C, Haidt J, Sawchuk CN. Specificity of disgust domains in the prediction of contamination anxiety and avoidance: A multimodal examination. Behavior Therapy. 2014; 45(4):469-81. doi: 10.1016/j.beth.2014.02.006

[29] Zanjani Z, Yaghubi H, Fata L, Shairi M, Gholami Fesharaki M. [Psychometric properties of disgust propensity and sensitivity scale in Iranian sample (Persian)]. Clinical Psychology \& Personality. 2016; In press. 
[30] Baron RM, Kenny DA. The moderator-mediator variable distinction in social psychological research: Conceptual, strategic, and statistical considerations. Journal of Personality and Social Psychology. 1986; 51(6):1173-82. doi: 10.1037//00223514.51.6.1173

[31] Curtis V, Aunger R, Rabie T. Evidence that disgust evolved to protect from risk of disease. Proceedings of the Royal Society B: Biological Sciences. 2004; 271(Suppl 4):S131-S133. doi: 10.1098/ rsbl.2003.0144

[32] Oaten M, Stevenson RJ, and Case TI. Disgust as a diseaseavoidance mechanism. Psychological Bulletin. 2009; 135(2):30321. doi: $10.1037 / \mathrm{a} 0014823$

[33] Fisher PL, Wells A. How effective are cognitive and behavioral treatments for obsessive-compulsive disorder? A clinical significance analysis. Behaviour Research and Therapy. 2005; 43(12):1543-58. doi: 10.1016/j.brat.2004.11.007 